\title{
Ventilator-associated respiratory infection following lung transplantation
}

\author{
Jordi Riera ${ }^{1}$, Berta Caralt ${ }^{1}$, Iker López ${ }^{2}$, Salvador Augustin ${ }^{3}$, Antonio Roman ${ }^{4,5}$, \\ Joan Gavalda ${ }^{6,7}$, Jordi Rello ${ }^{1,5}$ and the Vall d'Hebron Lung Transplant \\ Study Group ${ }^{8}$
}

\begin{abstract}
Affiliations: 'Dept of Critical Care, Vall d'Hebron University Hospital, Vall d'Hebron Research Institute, Universitat Autònoma de Barcelona, Barcelona, Spain. ${ }^{2}$ Dept of Thoracic Surgery, Vall d'Hebron University Hospital, Vall d'Hebron Research Institute, Universitat Autònoma de Barcelona, Barcelona, Spain. ${ }^{3}$ Liver Unit, Dept of Internal Medicine, Vall d'Hebron University Hospital, Vall d'Hebron Research Institute, Universitat Autònoma de Barcelona, Barcelona, Spain. 'Dept of Pneumology, Vall d'Hebron University Hospital, Vall d'Hebron Research Institute, Universitat Autònoma de Barcelona, Barcelona, Spain. ${ }^{5}$ Centro de Investigación Biomédica en Red de Enfermedades Respiratorias (CIBERES), Instituto de Salud Carlos III, Madrid, Spain. ${ }^{6}$ Dept of Infectious Diseases, Vall d'Hebron University Hospital, Vall d'Hebron Research Institute, Universitat Autònoma de Barcelona, Barcelona, Spain. ${ }^{7}$ Red Española de Investigación de Patologías Infecciosas (REIPI), Instituto de Salud Carlos III, Madrid, Spain. ${ }^{8}$ For a full list of the Vall d'Hebron Lung Transplant Study Group investigators see the Acknowledgements section.
\end{abstract}

Correspondence: Jordi Riera, Dept of Critical Care, Vall d'Hebron University Hospital, Vall d'Hebron Research Institute, Universitat Autònoma de Barcelona, Paseo Vall d'Hebron 119-129, 08035 Barcelona, Spain.

E-mail: jorrieraQuvhebron.net

ABSTRACT The medical records of 170 adult patients who underwent lung transplantation between January 2010 and December 2012 were reviewed to assess the incidence, causative organisms, risk factors and outcomes of post-operative pneumonia and tracheobronchitis.

$20(12 \%)$ patients suffered 24 episodes of ventilator-associated pneumonia. The condition was associated with mean increases of 43 days in mechanical ventilation and of 35 days in hospital stay, and significantly higher hospital mortality (OR 9.0, 95\% CI 3.2-25.1). Pseudomonas aeruginosa (eight out of 12 patients were multidrug-resistant) was the most common pathogen, followed by Enterobacteriaceae (one out of five patients produced extended-spectrum $\beta$-lactamases). Gastroparesis occurred in 55 (32\%) patients and was significantly associated with pneumonia (OR 6.2, 95\% CI 2.2-17.2). Ventilator-associated tracheobronchitis was associated with a mean increase of 28 days in mechanical ventilation and 30.5 days in hospital stay, but was not associated with higher mortality (OR 1.2, 95\% CI 0.4-3.2). Pseudomonas aeruginosa (six out of 16 patients were multidrug resistant) was the most common pathogen, followed by Enterobacteriaceae (three out of 14 patients produced extended-spectrum $\beta$-lactamase). Patients with gastroparesis also had more episodes of ventilator-associated tracheobronchitis ( $40 \%$ versus $12 \%, \mathrm{p}<0.001)$.

In conclusion, ventilator-associated pneumonia following lung transplantation increased mortality. Preventing gastroparesis probably decreases the risk of pneumonia and tracheobronchitis. Multidrugresistant bacteria frequently cause post-lung-transplantation pneumonia and tracheobronchitis.

@ERSpublications

VAP following lung transplantation increases mortality and gastroparesis increases the incidence of VAP http://ow.ly/CeRgp

Received: May 232014 | Accepted after revision: Sept 012014 | First published online: Oct 302014

Support statement: This study was partly supported by Instituto de Salud Carlos III, Madrid, Spain (grant FIS 11/01122) and by unrestricted grants from Air Liquide (Madrid, Spain) and the PCI Pneumonia project of Centro de Investigación Biomédica en Red de Enfermedades Respiratorias (CIBERES; Madrid).

Conflict of interest: Disclosures can be found alongside the online version of this article at erj.ersjournals.com

Copyright OERS 2015 


\section{Introduction}

Lung transplantation has become a standard of care for selected patients with end-stage non-malignant disabling lung disease [1]. Post-lung-transplant survival has improved markedly in recent years, but due to a variety of complications mortality remains higher than in other solid organ transplants, such as heart, kidney or liver. Infection is among the most common complications and is associated with high mortality rates [2]. Certain specific circumstances make the lung graft highly vulnerable to respiratory infection [3]. Donor conditions, such as decreased consciousness and mechanical ventilation, increase the risk of bacterial colonisation and infection, which may be subclinical. In addition, recipients are often colonised prior to transplant by pathogens that may be reactivated after surgery. Contact of the graft with the environment, inadequate lymphatic drainage and the effects of nerve damage during transplantation on airway clearance all increase the risk of lung infection. Post-operative gastroparesis can delay gastric emptying and thereby increase the risk of gastro-oesophageal reflux, aspiration and respiratory infection $[4,5]$. In addition, ventilator-associated tracheobronchitis (VAT) and ventilator-associated pneumonia (VAP) may complicate the post-operative course and may have severe consequences in this highly immunosuppressed population. In the past few years, protocols for immunosuppression and antibiotic prophylaxis have been developed [6] and antimicrobial resistance has increased [7], and all this may have changed the impact of respiratory infections on the outcomes of lung transplant recipients. There are also inconsistencies in the definitions of pneumonia and tracheobronchitis, which may partly explain the conflicting results between studies of the impact of post-lung-transplant respiratory infection [3, 8-10].

Our hypothesis for the present study was that post-lung-transplant pneumonia and tracheobronchitis are associated with worse outcomes. The primary objective was to assess their impact on intensive care unit (ICU) and hospital length of stay, and on survival. Secondary objectives were to identify their causative organisms and their potential risk factors.

\section{Methods}

The study took place in the ICU of the Vall d'Hebron University Hospital (Barcelona, Spain). It is a mixed medical and surgical unit with 32 beds in a tertiary hospital with 1100 beds. The study was approved by the ethics committee of the hospital (study PR_AG_279-2013). We retrospectively reviewed the medical records of consecutive adult patients who were admitted to the ICU after lung transplantation between January 2010 and December 2012. We assessed hospital stay, survival, pathogens and risk factors. The follow-up period ended 1 year after ICU admission. The ethics committee considered that informed consent of the patients was not necessary due to the retrospective and non-interventional nature of the study.

\section{Lung transplant preparation, procedure and post-operative care}

Lungs were obtained via en bloc extraction and immediately immersed in a solution for rapid cooling, transport and storage (Perfadex; Vitrolife, Gothenburg, Sweden). Single lung transplants were performed via anterior thoracotomy and double lung transplants via bilateral anterior thoracotomy with transverse sternotomy (clamshell approach). Prior to lung reperfusion, $500 \mathrm{mg}$ of intravenous methylprednisolone was administered. Extracorporeal circulation was only used if respiratory or haemodynamic instability might impede the lung transplant procedure.

Antibiotic prophylaxis comprised of ceftazidime and amoxicillin-clavulanate for 5 days. Microorganism-based surveillance of patients on the waiting list was undertaken before surgery. If any microorganism was isolated prior to surgery, appropriate post-operative antibiotic therapy was selected. Nebulised amphotericin and i.v. ganciclovir were given as prophylaxis against Aspergillus species and Cytomegalovirus, respectively. Pneumocystis prophylaxis with trimethoprim-sulfamethoxazole was started on post-operative day 30. The general VAP prophylaxis consisted of head-of-bed elevation, and selective oropharyngeal and digestive-tract decontamination with tobramycin and colistin. Subglottic drainage tubes were not used.

During the first few days following lung transplantation, patients were monitored via a Swan-Ganz heart catheter. Negative fluid balance was carefully maintained to avoid lung oedema and maintain adequate renal perfusion. Lung protective mechanical ventilation with low tidal volumes $\left(<7 \mathrm{~mL} \cdot \mathrm{kg}^{-1}\right.$ of ideal body weight) and airway pressures (plateau pressure $<30 \mathrm{cmH}_{2} \mathrm{O}$ ) was scheduled with low doses of sedatives (target Ramsay sedation scale of 2) and early extubation if the patient was stable. A 30-min T-tube test was always performed before extubation. If the first test failed, sedation was resumed and a daily $30 \mathrm{~min}$ T-tube trial was started. When the patient failed the test repeatedly or had to be re-intubated, early tracheostomy was performed. If diaphragmatic paresis was suspected, thoracic echography was performed to evaluate the diaphragm movement. Physiotherapy and early mobilisation were started as soon as the patient was stabilised. If refractory hypoxaemia developed, the patient was placed in the prone position to optimise oxygenation. If pulmonary hypertension developed, nitric oxide (5-20 ppm) was added to the 
inhaled gas. No extracorporeal support was used in these cases. Enteral feeding was usually started on the second post-operative day. If gastroparesis was diagnosed, the patient received parenteral feeding and gastric pro-kinetic drugs. Routine pre-operative evaluation of gastroparesis was not performed.

No induction immunosuppression was used. Tacrolimus (serum target range 10-15 ng. $\mathrm{mL}^{-1}$ ) and methylprednisolone $\left(1 \mathrm{mg} \cdot \mathrm{kg}^{-1} \cdot \mathrm{day}^{-1}\right.$ for the first 5 days followed by $0.3 \mathrm{mg} \cdot \mathrm{kg}^{-1} \cdot \mathrm{day}^{-1}$ ) were used as standard immediate post-operative immunosuppressant. On post-operative day five mycophenolate (1-2 g per day) was started. If acute cellular rejection was diagnosed, a 3-day course of high-dose i.v. methylprednisolone $\left(5-10 \mathrm{mg} \cdot \mathrm{kg}^{-1} \cdot \mathrm{day}^{-1}\right)$ was administered. If antibody-mediated rejection was diagnosed, treatment with plasmapheresis, i.v. immunoglobulin and rituximab was started.

\section{Data collection and definitions}

Data were compiled from the hospital's patient data management system.

Pneumonia was defined as new or progressive radiographic opacity, $\geq 10^{4} \mathrm{CFU} \cdot \mathrm{mL}^{-1}$ in bronchoalveolar lavage (BAL) fluid and at least two of the following: fever $>38^{\circ} \mathrm{C}$, leukocytosis $\left(\geq 15000 \mathrm{cells} \cdot \mathrm{mm}^{-3}\right)$ or leukopenia $\left(<4000\right.$ cells $\left.\cdot \mathrm{mm}^{-3}\right)$ and purulent secretions. Late-onset pneumonia was defined when it occurred after $>4$ days of admission [11]. When associated with mechanical ventilation, it was defined as VAP. When VAP was suspected, a fibrobronchoscopy was performed and BAL was obtained. Galactomannan in the BAL was always measured and Aspergillus was investigated using cultures and PCR. No routine surveillance for sampling patients was implemented.

Tracheobronchitis was defined as fever $>38^{\circ} \mathrm{C}$, new or increased sputum production, $\geq 10^{6} \mathrm{CFU} \cdot \mathrm{mL}^{-1}$ in sputum suctioned from the endotracheal tube, and no new pulmonary opacities on chest radiographs [12]. When associated with mechanical ventilation, it was defined as VAT.

Multidrug-resistant Pseudomonas aeruginosa was defined as resistance to at least three of the following antibiotics classes: antipseudomonal penicillins, antipseudomonal oxyimino- $\beta$-lactams, fluoroquinolones, aminoglycosides and carbapenems [13]. Enterobacteriaceae producing extended-spectrum $\beta$-lactamase were analysed as a subgroup [14].

Phrenic paresis was defined as difficulty in weaning from the ventilator and echographic signs of paresis. An echogram of diaphragm function was performed at $3.5-5.0 \mathrm{MHz}$, with a $10 \mathrm{MHz}$ phased-array probe [15]. Diaphragm excursion was evaluated by displacement in the $M$ mode. The lower limit of diaphragm excursion was considered to be $9 \mathrm{~mm}$ in females and $10 \mathrm{~mm}$ in males. The thickening fraction was calculated with the $\mathrm{M}$ mode, as follows.

Thickening fraction $=($ thickness at end-inspiration - thickness at end-expiration $) /$ thickness at end-expiration

A thickening fraction $>20 \%$ was considered normal. Qualitative discriminations were made between reduced and paradoxical inspiratory movement [15].

Gastroparesis was defined as the presence of three criteria: 1) abdominal discomfort with enteral nutrition; 2) gastric-contents aspiration volume (measured every $6 \mathrm{~h}$ ) $>500 \mathrm{~mL}$ or $150-500 \mathrm{~mL}$ at two consecutive measurements [16]; and stomach dilation on radiographs. All patients with gastroparesis received gastric pro-kinetic drugs.

\section{Statistical analysis}

Continuous data are reported as median (interquartile range (IQR)). Categorical data are reported as $\mathrm{n}(\%)$. Differences between categorical variables were assessed with the Chi-squared test or Fisher's exact test, as appropriate. Continuous variables were compared with the t-test or the Mann-Whitney test, as appropriate. A two-sided $\mathrm{p}<0.05$ was considered statistically significant. Multivariate analysis for pneumonia was conducted via stratified analysis and logistic regression. Variables included in the analysis were selected based on previous bibliography, avoiding over fitting (thus limiting the inclusion of one variable for each five to 10 cases), and taking into account the exploratory results of the univariate analysis. All analyses were performed with statistical software (PASW Statistics 19.0; SPSS, Chicago, IL, USA).

\section{Results}

Patients

A total of 170 consecutive lung transplants were assessed (table 1). The median (IQR) duration of mechanical ventilation was $12.5(2.0-37.2)$ days and 5 (2.0-25.0) days for the patients without phrenic paresis. The median (IQR) ICU stay and hospital stay were 18.0 (6.0-38.7) days and 40.0 (28.0-60.0) days, respectively. $17(10 \%)$ patients died in the ICU and hospital mortality was $15 \% .18(11 \%)$ patients were prioritised for urgent transplantation, and eight (5\%) were on mechanical ventilation prior to lung 


\section{TABLE 1 Characteristics and global outcomes of the population}

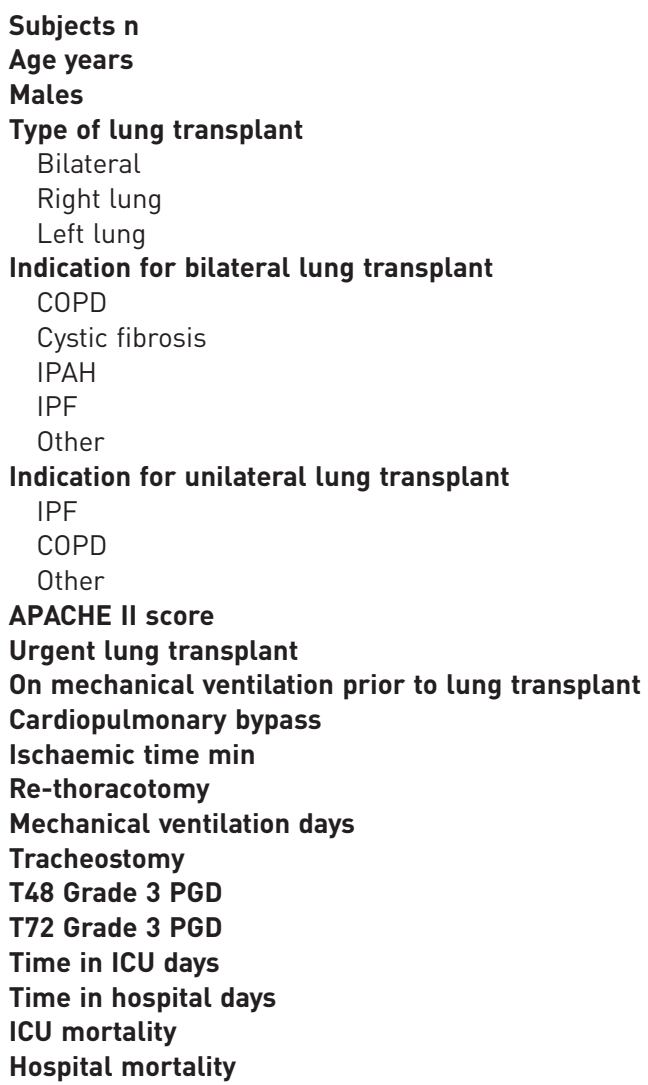

Data are presented as median (interquartile range) or $\mathrm{n}(\%)$, unless otherwise stated. COPD: chronic obstructive pulmonary disease; IPAH: idiopathic pulmonary arterial hypertension; IPF: idiopathic pulmonary fibrosis; APACHE: Acute Physiology and Chronic Health Evaluation; PGD: primary graft dysfunction; ICU: intensive care unit; T48: 48 h from ICU admission; T72: $72 \mathrm{~h}$ from ICU admission.

transplant. 40.6\% of the cohort had an Acute Physiology and Chronic Health Evaluation II score between 20 and 30.

\section{Incidence of respiratory infections}

All the episodes developed in mechanically ventilated patients. There were 24 episodes of VAP in 20 (12\%) patients. 21 episodes were late onset and the median (IQR) onset was 33 (13-46) days after lung transplant. Other details of these episodes are summarised in table 2. There were 55 episodes of VAT in 36 (21\%) patients and the median onset was 25 (12-37) days. Six patients (five of them with gastroparesis) suffered episodes of both VAT and VAP. There were 5.2 episodes of VAP and 11.7 episodes of VAT per 1000 ventilator-days.

Figure 1 shows the flowchart of all the screened patients that developed any new lung infiltrates not related to primary graft dysfunction. Four necropsies were performed in the 14 patients with noninfectious pulmonary opacities that died. Signs of humoral rejection, thromboembolism, acute respiratory distress syndrome and pulmonary haemorrhage were reported.

\section{Risk factors for respiratory infections}

$60(35 \%)$ patients had phrenic paresis and 55 (32\%) had gastroparesis. Gastroparesis was more common in patients who had undergone bilateral transplantation than in those who had undergone unilateral transplant ( $40 \%$ versus $22 \%, \mathrm{p}=0.01$ ). The same was true for phrenic paresis $(31 \%$ versus $25 \%, \mathrm{p}=0.04)$. Both gastroparesis and phrenic paresis were associated with more days of ventilation and longer ICU and hospital stay (table 3). Compared with the rest of the population, patients with gastroparesis had more episodes of VAT $(40 \%$ versus $12 \%, \mathrm{p}<0.001)$ and VAP $(26 \%$ versus $5 \%, \mathrm{p}<0.001)$. Similarly, patients with 
TABLE 2 Features of the 24 episodes of ventilator-associated pneumonia

\begin{tabular}{|c|c|c|c|c|c|c|c|c|c|c|c|c|c|c|c|c|}
\hline \multirow[t]{2}{*}{ Patient } & \multirow[t]{2}{*}{$\begin{array}{l}\text { Age } \\
\text { years }\end{array}$} & \multirow[t]{2}{*}{$\begin{array}{l}\text { Lung } \\
\text { disease }\end{array}$} & \multirow[t]{2}{*}{$\begin{array}{l}\text { Lung } \\
\text { transplantation }\end{array}$} & \multicolumn{2}{|c|}{$\begin{array}{l}\text { Cultures prior to lung } \\
\text { transplantation }\end{array}$} & \multirow[t]{2}{*}{$\begin{array}{l}\text { Day of } \\
\text { diagnosis }\end{array}$} & \multirow[t]{2}{*}{ Microorganism } & \multirow[t]{2}{*}{$\begin{array}{l}\text { Antibiotics at } \\
\text { diagnosis }\end{array}$} & \multirow{2}{*}{$\begin{array}{l}\text { Leukocytes } \\
\times 10^{9} \\
\text { cells: } L^{-1}\end{array}$} & \multirow[t]{2}{*}{$\begin{array}{l}\text { Temperature } \\
{ }^{\circ} \mathrm{C}\end{array}$} & \multirow[t]{2}{*}{$\mathrm{FiO}_{2}$} & \multirow[t]{2}{*}{$\begin{array}{l}\text { Chest } \\
\text { radiograph }\end{array}$} & \multirow[t]{2}{*}{$\begin{array}{l}\text { Purulent } \\
\text { secretions }\end{array}$} & \multirow{2}{*}{$\begin{array}{l}\text { Time to } \\
\text { death } \\
\text { days }\end{array}$} & \multirow[t]{2}{*}{ Gastroparesis } & \multirow{2}{*}{$\begin{array}{l}\text { Most relevant } \\
\text { abdominal symptoms/ } \\
\text { signs related with } \\
\text { gastroparesis }\end{array}$} \\
\hline & & & & Donor & Recipient & & & & & & & & & & & \\
\hline \multirow[t]{2}{*}{1} & 59 & COPD & Bilateral & & & 21 & E. cloacae ESBL & None & $4-15$ & $>38$ & 0.6 & $2 / 4$ & Yes & & Yes & $\begin{array}{l}\text { Abdominal distension and } \\
\text { pain, CT scan with } \\
\text { stomach dilation }\end{array}$ \\
\hline & & & & & & 52 & $P$. aeruginosa & None & 4-15 & $>38$ & 1 & $2 / 4$ & Yes & & & \\
\hline 2 & 59 & COPD & Bilateral & & S. maltophilia & 16 & $\begin{array}{l}\text { Multi-resistant } \\
\text { P. aeruginosa and } \\
\text { E. cloacae }\end{array}$ & None & 4-15 & $>38$ & 0.3 & $1 / 4$ & Yes $^{\#}$ & & Yes & $\begin{array}{l}\text { Vomiting, stomach } \\
\text { dilation on radiograph }\end{array}$ \\
\hline 3 & 62 & COPD & Bilateral & S. aureus & & 12 & $\begin{array}{l}\text { Multi-resistant } \\
\text { P. aeruginosa }\end{array}$ & None & $>15$ & $>38$ & 0.5 & $1 / 4$ & Yes & & Yes & $\begin{array}{l}\text { Vomiting and abdominal } \\
\text { pain, stomach dilation on } \\
\text { radiograph }\end{array}$ \\
\hline 4 & 50 & COPD & Bilateral & & & 28 & $\begin{array}{l}\text { Multi-resistant } \\
\text { P. aeruginosa }\end{array}$ & Meropenem & $>15$ & $>38$ & 0.7 & $3 / 4$ & Yes & 33 & Yes & $\begin{array}{l}\text { Abdominal distension, } \\
\text { stomach dilation on } \\
\text { radiograph }\end{array}$ \\
\hline 5 & 55 & COPD & Bilateral & K. pneumoniae & & 19 & A. fumigatus & $\begin{array}{c}\text { Pipe-tazo } \\
\text { Nebulised colistin }\end{array}$ & $>15$ & $<38$ & 0.5 & $1 / 4$ & Yes & 162 & No & \\
\hline 6 & 49 & COPD & Bilateral & & $P$. aeruginosa & 15 & Unknown? & $\begin{array}{l}\text { Cefepime } \\
\text { Nebulised colistin }\end{array}$ & $>15$ & $<38$ & 0.4 & $1 / 4$ & Yes $^{+}$ & & Yes & $\begin{array}{l}\text { Abdominal distension and } \\
\text { pain, CT scan with } \\
\text { stomach dilation }\end{array}$ \\
\hline 7 & 64 & COPD & Bilateral & & & 44 & $\begin{array}{l}\text { Multi-resistant } \\
\text { P. aeruginosa }\end{array}$ & None & $>15$ & $<38$ & 0.4 & $1 / 4$ & Yes $^{\#}$ & 190 & Yes & $\begin{array}{c}\text { Abdominal distension, } \\
\text { CT scan with intestinal } \\
\text { dilation }\end{array}$ \\
\hline 8 & 57 & COPD & Bilateral & & & 65 & Unknown" & None & $4-15$ & $>38$ & 0.4 & $1 / 4$ & Yes $^{+}$ & & No & \\
\hline 9 & 45 & COPD & Bilateral & & & 21 & MRSA & None & $4-15$ & $>38$ & 0.6 & $2 / 4$ & Yes & & Yes & $\begin{array}{l}\text { Abdominal distension, } \\
\text { diarrhoea and stomach } \\
\text { dilation on radiograph }\end{array}$ \\
\hline \multirow[t]{3}{*}{10} & 56 & COPD & Right & S. aureus & & 16 & $P$. aeruginosa & $\begin{array}{l}\text { Ceftazidime } \\
\text { Amoxi-clav }\end{array}$ & 4-15 & $>38$ & 0.6 & $2 / 4^{\S}$ & Yes & 81 & Yes & $\begin{array}{l}\text { Abdominal distension, } \\
\text { stomach dilation on } \\
\text { radiograph }\end{array}$ \\
\hline & & & & & & 31 & S. maltophilia & $\begin{array}{l}\text { Tigecycline } \\
\text { Nebulised colistin }\end{array}$ & $4-15$ & $>38$ & 1 & $3 / 4^{f}$ & Yes & & & \\
\hline & & & & & & 70 & $\begin{array}{l}\text { Multi-resistant } \\
\text { P. aeruginosa }\end{array}$ & $\begin{array}{l}\text { Meropenem } \\
\text { Nebulised colistin }\end{array}$ & $4-15$ & $>38$ & 0.6 & $3 / 4^{f}$ & Yes & & & \\
\hline
\end{tabular}




\begin{tabular}{|c|c|c|c|c|c|c|c|c|c|c|c|c|c|c|c|c|}
\hline \multirow[t]{2}{*}{ Patient } & \multirow[t]{2}{*}{$\begin{array}{l}\text { Age } \\
\text { years }\end{array}$} & \multirow[t]{2}{*}{$\begin{array}{l}\text { Lung } \\
\text { disease }\end{array}$} & \multirow[t]{2}{*}{$\begin{array}{c}\text { Lung } \\
\text { transplantation }\end{array}$} & \multicolumn{2}{|c|}{$\begin{array}{l}\text { Cultures prior to lung } \\
\text { transplantation }\end{array}$} & \multirow[t]{2}{*}{$\begin{array}{l}\text { Day of } \\
\text { diagnosis }\end{array}$} & \multirow[t]{2}{*}{ Microorganism } & \multirow[t]{2}{*}{$\begin{array}{l}\text { Antibiotics at } \\
\text { diagnosis }\end{array}$} & \multirow{2}{*}{$\begin{array}{l}\text { Leukocytes } \\
\times 10^{9} \\
\text { cells } \cdot \mathrm{L}^{-1}\end{array}$} & \multirow[t]{2}{*}{$\begin{array}{l}\text { Temperature } \\
{ }^{\circ} \mathrm{C}\end{array}$} & \multirow[t]{2}{*}{$\mathrm{FiO}_{2}$} & \multirow[t]{2}{*}{$\begin{array}{l}\text { Chest } \\
\text { radiograph }\end{array}$} & \multirow[t]{2}{*}{$\begin{array}{c}\text { Purulent } \\
\text { secretions }\end{array}$} & \multirow{2}{*}{$\begin{array}{l}\text { Time to } \\
\text { death } \\
\text { days }\end{array}$} & \multirow[t]{2}{*}{ Gastroparesis } & \multirow{2}{*}{$\begin{array}{l}\text { Most relevant } \\
\text { abdominal symptoms/ } \\
\text { signs related with } \\
\text { gastroparesis }\end{array}$} \\
\hline & & & & Donor & Recipient & & & & & & & & & & & \\
\hline 11 & 18 & $\mathrm{CF}$ & Bilateral & $\begin{array}{l}\text { Aspergillus } \\
\text { spp. }\end{array}$ & $\begin{array}{l}\text { S. maltophilia } \\
\text { and MRSA }\end{array}$ & 15 & P. aeruginosa & Pipe-tazo & $>15$ & $<38$ & 0.5 & $1 / 4$ & Yes & & Yes & $\begin{array}{l}\text { Vomiting and abdominal } \\
\text { pain, stomach dilation on } \\
\text { radiograph }\end{array}$ \\
\hline 12 & 62 & $\begin{array}{c}\text { Histiocytosis } \\
\mathrm{X}\end{array}$ & Bilateral & M. tuberculosis & $\begin{array}{l}\text { S. aureus and } \\
\text { Haemophilus } \\
\text { spp. }\end{array}$ & 13 & Unknown" & Anti-TB drugs & $>15$ & $<38$ & 0.8 & $1 / 4$ & Yes & & Yes & $\begin{array}{l}\text { Nausea and vomiting, } \\
\text { abdominal distension }\end{array}$ \\
\hline 13 & 57 & LAM & Bilateral & S. aureus & S. aureus & 0 & S. aureus & $\begin{array}{l}\text { Amoxi-clav } \\
\text { Aztreonam }\end{array}$ & $>15$ & $>38$ & 0.5 & $2 / 4$ & Yes & 8 & No & \\
\hline 14 & 46 & IPAH & Bilateral & S. aureus & & 13 & Unknown? & $\begin{array}{l}\text { Cefepime } \\
\text { Nebulised colistin }\end{array}$ & $>15$ & $<38$ & 0.7 & $1 / 4$ & Yes & & Yes & $\begin{array}{l}\text { Abdominal distension, } \\
\text { diarrhoea, stomach } \\
\text { dilation on radiograph }\end{array}$ \\
\hline \multirow[t]{2}{*}{15} & 46 & IPF & Bilateral & S. aureus & $\begin{array}{l}\text { Multi-resistant } \\
\text { P. aeruginosa }\end{array}$ & 55 & $\begin{array}{l}\text { Multi-resistant } \\
\text { P. aeruginosa }\end{array}$ & Nebulised colistin & $>15$ & $<38$ & 0.5 & $2 / 4$ & Yes & 133 & Yes & $\begin{array}{c}\text { Vomiting and abdominal } \\
\text { pain }\end{array}$ \\
\hline & & & & & & 106 & $\begin{array}{l}\text { Multi-resistant } \\
\text { P. aeruginosa }\end{array}$ & $\begin{array}{c}\text { Amikacine } \\
\text { Nebulised colistin }\end{array}$ & $4-15$ & $>38$ & 0.5 & $1 / 4$ & Yes & & & \\
\hline 16 & 65 & IPF & Left & C. freundii & & 33 & $\begin{array}{l}\text { K. pneumoniae and } \\
\text { E. cloacae }\end{array}$ & None & $>15$ & $<38$ & 0.7 & $2 / 4^{\S}$ & Yes & & Yes & $\begin{array}{c}\text { Abdominal distension, } \\
\text { diarrhoea }\end{array}$ \\
\hline 17 & 65 & IPF & Left & $\begin{array}{l}\text { Haemophilus } \\
\text { spp. }\end{array}$ & & 164 & $\begin{array}{l}\text { Multi-resistant } \\
\text { P. aeruginosa }\end{array}$ & Nebulised colistin & $>15$ & $<38$ & 0.5 & $2 / 4^{\S}$ & Yes & 171 & Yes & $\begin{array}{l}\text { Nausea and abdominal } \\
\text { distension, diarrhoea, CT } \\
\text { scan with stomach and } \\
\text { colic dilation }\end{array}$ \\
\hline 18 & 49 & IPF & Right & S. aureus & S. aureus & 0 & S. aureus & None & $<4$ & $<38$ & 0.8 & $1 / 4^{\# \#}$ & Yes & 42 & Yes & $\begin{array}{l}\text { Abdominal distension, СT } \\
\text { scan with stomach and } \\
\text { gallbladder dilation }\end{array}$ \\
\hline 19 & 31 & CLAD & Right & & & 0 & E. aerogenes & None & $>15$ & $>38$ & 0.5 & $1 / 4^{\S}$ & $\mathrm{No}^{+}$ & & No & \\
\hline 20 & 46 & IPF & Left & $\begin{array}{l}\text { E. coli and } \\
\text { S. pneumoniae }\end{array}$ & & 5 & P. aeruginosa & Cefotaxime & $>15$ & $<38$ & 0.7 & $1 / 4^{\S}$ & Yes & 26 & No & \\
\hline
\end{tabular}

Chest radiography data is presented as quadrants with opacities. FlO 2 : inspiratory oxygen fraction; COPD: chronic obstructive pulmonary disease; ESBL: extended-spectrum $\beta$-lactamase; CT: computed tomography; Pipe-tazo: piperacillin-tazobactam; Amoxi-clav: amoxicillin-clavulanate; CF: cystic fibrosis; TB: tuberculosis; LAM: lymphangioleiomyomatosis; IPAH: idiopathic pulmonary arterial hypertension; IPF: idiopathic pulmonary fibrosis; CLAD: chronic lung allograft dysfunction; E. cloacae: Enterobacter cloacae; P. aeruginosa: Pseudomonas aeruginosa; S. maltophilia: Stenotrophomonas maltophilia; S. aureus: Staphylococcus aureus; K. pneumoniae: Klebsiella pneumoniae; A. fumigatus: Aspergillus fumigatus; MRSA: methicillin-resistant S. aureus; M. tuberculosis: Mycobacterium tuberculosis; C. freundii: Citrobacter freundii; E. aerogenes: Enterobacter aerogenes; E. coli: Escherichia coli; S. pneumoniae: Streptococcus pneumoniae. ${ }^{\#}$ : dyspnoea; ${ }^{\text {": }}$ no histopathological evidence of acute rejection; ${ }^{+}$: rales; ${ }^{\S}$ : native quadrants; ${ }^{f}$ : native and graft quadrants; ${ }^{\# \#}$ : graft quadrants. 
phrenic paresis had more episodes of VAT $(32 \%$ versus $15 \%, \mathrm{p}<0.001)$ and showed a trend toward more episodes of VAP ( $18 \%$ versus $8 \%, \mathrm{p}=0.050)$.

Neither donor nor recipient colonisation was significantly associated with a higher incidence of ventilator-associated respiratory infection (OR 1.9 (95\% CI $0.8-4.3$ ) and OR 1.9 (95\% CI 0.8-4.9), respectively), regardless of type of transplantation (unilateral or bilateral) (table 4).

None of the underlying diseases were associated with the development of VAP or VAT. The 13 patients with cystic fibrosis were colonised before lung transplantation, but their incidence of ventilator-associated respiratory infection was similar to the rest of the population (23\% of VAT and $7.6 \%$ of VAP). Mechanical ventilation prior to transplantation was not associated with a higher risk of post-operative pneumonia ( $25 \%$ versus $11 \%, \mathrm{p}=0.23$ ) or tracheobronchitis ( $22 \%$ versus $13 \%, \mathrm{p}>0.99)$.

When gastroparesis, phrenic paresis and recipient and donor colonisation were included in a one-step multivariate logistic regression, only gastroparesis was an independent risk factor for VAP (OR 6.2, 95\% CI 2.2-17.2), and when this analysis was adjusted for days of mechanical ventilation the association was still significant (OR 3.1, 95\% CI 1.1-9.5; $\mathrm{p}=0.04$ ).

\section{Pathogens}

The top three pathogens in the patients with VAP were Pseudomonas aeruginosa (eight out of 12 patients were multidrug-resistant), followed by Enterobacteriaceae (one out of five patients produced extended-spectrum $\beta$-lactamase) and Staphylococcus aureus (one out of three patients were methicillin resistant) (fig. 2). P. aeruginosa (six out of 16 patients were multidrug-resistant) was also the most frequent pathogen in patients with VAT, followed by Enterobacteriaceae (three out of 14 patients produced extended-spectrum $\beta$-lactamase) and Staphylococcus aureus (one out of eight patients was methicillin resistant) (fig. 3). There were no documented episodes of viral pneumonitis.

\section{Patient outcomes}

VAP was significantly associated with longer mechanical ventilation $(+43$ days $)$, ICU stay $(+42.5$ days $)$ and hospital stay ( +35 days) (table 5$)$, as was VAT $(+28,+27.5$ and +30.5 days, respectively). VAP was also significantly associated with higher probability of ICU death (OR 10.4, 95\% CI 3.4-32) and hospital death (OR 9.0, 95\% CI 3.2-25.1). Figure 4 shows the Kaplan-Meier curve for survival in patients with VAP. In contrast, VAT was not associated with worse mortality (ICU death: OR 1.21, 95\% CI 0.4-3.2).

Gastroparesis was associated with a trend toward worse mortality in the ICU (OR 2.62, 95\% CI 0.95-7.21) and in the hospital (OR 1.80, 95\% CI 0.76-4.29), but phrenic paresis was not (table 3). No differences in mortality were found comparing single versus bilateral lung transplantation (13.5\% for single and $15.6 \%$ for bilateral; $\mathrm{p}>0.20$ ). The same was true when comparing patients aged $>55$ years versus the rest of the population $(15.4 \%$ and $14.1 \%$, respectively; $\mathrm{p}>0.20)$.

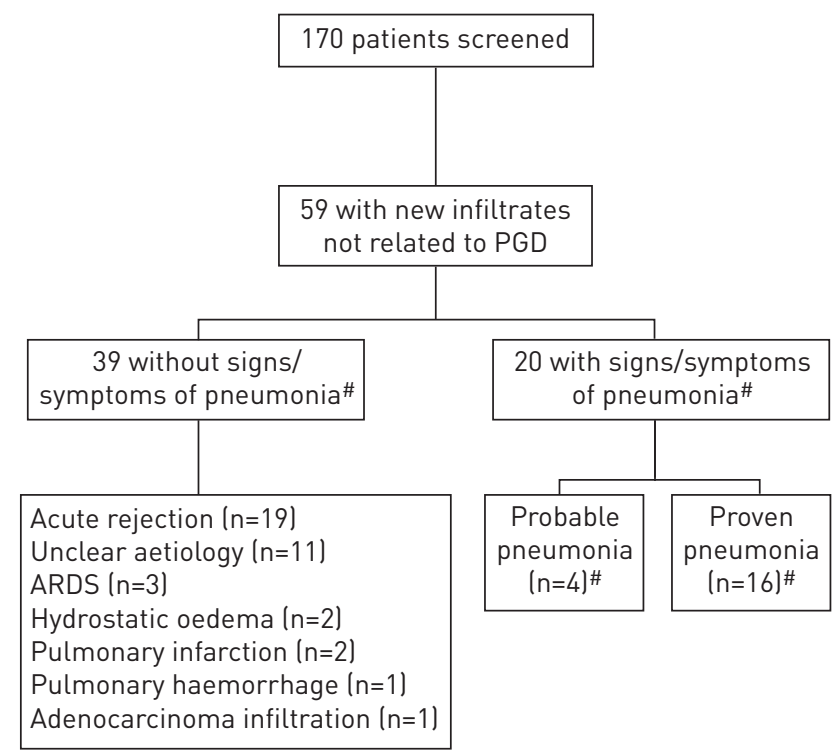

FIGURE 1 Flowchart of patients with new lung infiltrates not related to primary graft dysfunction (PGD). ARDS: acute respiratory distress syndrome. ${ }^{*}$ : following the International Society of Heart and Lung Transplantation criteria. 
TABLE 3 Outcomes of lung transplant recipients with or without gastroparesis or phrenic paresis

\begin{tabular}{|c|c|c|c|c|c|c|}
\hline & $\begin{array}{c}\text { With } \\
\text { gastroparesis }\end{array}$ & $\begin{array}{c}\text { Without } \\
\text { gastroparesis }\end{array}$ & p-value & $\begin{array}{l}\text { With phrenic } \\
\text { paresis }\end{array}$ & $\begin{array}{l}\text { Without phrenic } \\
\text { paresis }\end{array}$ & p-value \\
\hline Subjects $n$ & 55 & 115 & & 60 & 110 & \\
\hline Time in ICU days & $44(32-65)$ & $8(5-26.2)$ & $<0.001$ & $38(12-62)$ & $8(5-27)$ & $<0.001$ \\
\hline Time in hospital days & $64(45-80)$ & $31(24-48)$ & $<0.001$ & $60(37-80)$ & $32(25-48)$ & $<0.001$ \\
\hline Ventilator-associated tracheobronchitis & $22(40)$ & $14(12)$ & $<0.001$ & 19 (32) & $17(15)$ & $<0.001$ \\
\hline Hospital mortality & $11(20)$ & $14(12)$ & 0.12 & $9(15)$ & $16(15)$ & 0.94 \\
\hline
\end{tabular}

Data are presented as median (interquartile range) or $\mathrm{n}(\%)$, unless otherwise stated. ICU: intensive care unit.

\section{Discussion}

This is the first study to report a robust association between post-lung transplant VAP and ICU and hospital mortality. Both VAP and VAT increased costs, by increasing mechanical ventilation and ICU and hospital stays. Multidrug-resistant bacteria were common causative organisms of ventilator-associated respiratory infections. Interestingly, there was a strong association between gastroparesis and VAP, a finding that has obvious implications for prevention.

Previous studies found that the rate of infectious complications is higher in lung transplant recipients than in recipients of other solid organs, and that infections accounted for $>20 \%$ of mortality in the first 30 days following lung transplantation [9]. Bacterial pneumonia occurs most frequently in the early post-operative phase and, excluding primary graft dysfunction, is the major cause of new lung infiltrates in this period [17]. Nevertheless, attributable mortality is controversial [17-19]. In the present study we found a marked association between VAP and mortality. In contrast, a recent investigation of the clinical response to antimicrobial therapy in ICU patients with pneumonia (ventilator-associated in $77.1 \%$ of the cases) after lung or heart-lung transplantation found no impact of the condition on ICU mortality [10]. However, a recent meta-analysis reported attributable mortality associated with VAP $>60 \%$ in surgical patients [20] and larger previous studies in lung transplant recipients found that post-operative pneumonia significantly increased mortality $[3,9]$. The discrepancies between these studies may be attributed to differences in immunosuppression protocols, antibiotic regimens and microbiology patterns in the study centres. Moreover, there are no gold standard criteria for the definition of VAP [21], particularly in lung transplant recipients, and the differences in the definitions between the studies may be crucial in the interpretation of the results. We used the criteria of the American Thoracic Society/Infectious Diseases Society of America [11] which are divided into clinical, radiological and microbiological criteria, as in the definition proposed by the International Society of Heart and Lung Transplantation (ISHLT) consensus statement [22]. For the diagnosis of tracheobronchitis we also applied criteria that fulfil those proposed by the ISHLT. Using these criteria, a recently published prospective, observational study concluded that immunocompromised patients were at a higher risk for ventilator-associated respiratory infection likely to increase ICU and

TABLE 4 Pre-operative colonisation and ventilator-associated respiratory infection in lung transplant recipients

\begin{tabular}{lcccccc} 
& $\begin{array}{c}\text { Donor } \\
\text { colonised }\end{array}$ & $\begin{array}{c}\text { Donor not } \\
\text { colonised }\end{array}$ & p-value & $\begin{array}{c}\text { Recipient } \\
\text { colonised }\end{array}$ & $\begin{array}{c}\text { Recipient not } \\
\text { colonised }\end{array}$ & p-value \\
\hline $\begin{array}{l}\text { VAT } \\
\quad \text { Unilateral transplant }\end{array}$ & 17 & 21 & 0.73 & 20 & 19 & 0.91 \\
$\quad \begin{array}{l}\text { Bilateral transplant } \\
\text { VAP }\end{array}$ & 21 & 25 & 0.75 & 19 & 29 & 0.33 \\
$\quad$ Unilateral transplant & 6 & 3 & 0.52 & 0 & 4 & $>0.99$ \\
$\quad \begin{array}{l}\text { Bilateral transplant } \\
\text { Hospital mortality }\end{array}$ & 14 & 17 & 0.84 & 17 & 14 & $>0.99$ \\
\end{tabular}

Data are presented as \%, unless otherwise stated. VAT: ventilator-associated tracheobronchitis; VAP: ventilator-associated pneumonia. 


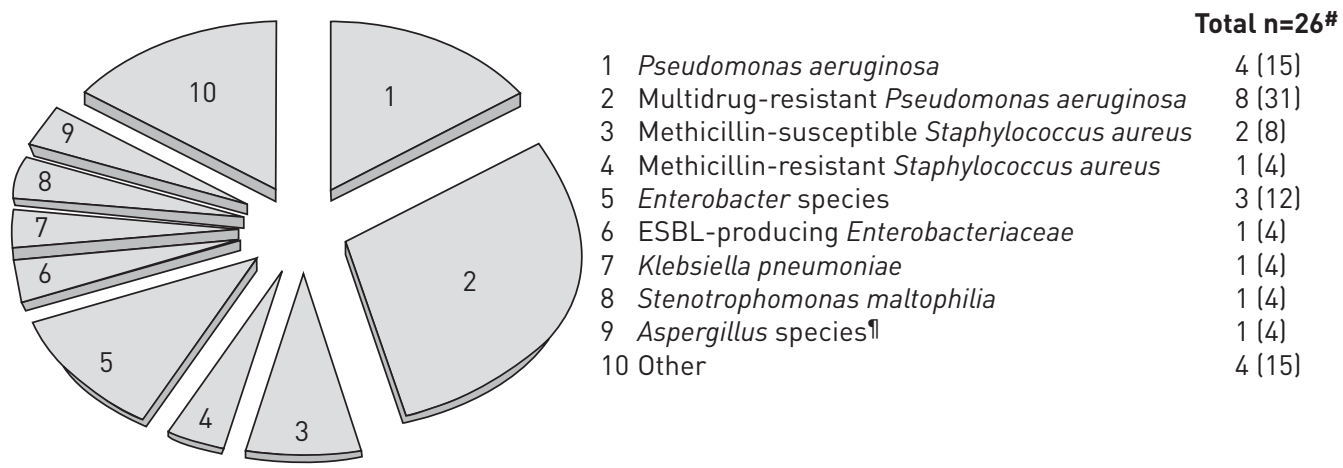

FIGURE 2 Pathogens in lung transplant recipients that caused ventilator-associated pneumonia. ESBL: extended-spectrum $\beta$-lactamase. ${ }^{*}$ : polymicrobial in two cases; ๆ: aspergilloma.

hospital length of stay [23]. Similarly, and in agreement with other studies [18, 24], we found VAT to be significantly associated with longer mechanical ventilation, and ICU and hospital stay, but not with mortality.

Multidrug-resistant bacteria are common in lung transplant recipients $[10,25]$. In our patients, multidrug-resistant $P$. aeruginosa was the most frequent microorganism in the patients with VAP, and was also common in those with VAT. Lung transplant recipients receive diverse antibiotics in the post-operative period, which may favour the development of multidrug-resistant pathogens. This fact should be taken into account in the choice of empiric antibiotic therapy for respiratory infection developing in this population.

Gastroparesis was associated with a higher incidence of VAP and worse ICU and hospital mortality. BERKOWITZ et al. [26] observed that obliterative bronchiolitis was more frequent in patients with gastroparesis than in nonsymptomatic patients. Curiously, those authors reported that no patients had symptoms immediately after surgery. In contrast, but in agreement with our findings, SMITH et al. [27] found 11 early abdominal complications in 75 lung transplant recipients, including four cases of nonobstructive ileus. This gastrointestinal hypodynamism is thought to be secondary to vagus nerve injury during surgery, and immunosuppression in a population with an above-average incidence of pre-operative oesophageal dysmotility [28]. These conditions promote gastro-oesophageal reflux, which increases the risk of aspiration in lung transplant recipients who also have impaired cough ability and impaired mucociliary clearance. Reflux and aspiration probably contribute to chronic lung dysfunction $[4,26,29,30]$, and anti-reflux surgery has been proposed in order to improve lung graft function $[5,31,32]$. In lung transplant and heart-lung transplant recipients there is an association between reflux and respiratory infections [33]. In 1990, ReiD et al. [4] recommended studying gastric emptying in heart-lung transplant recipients who

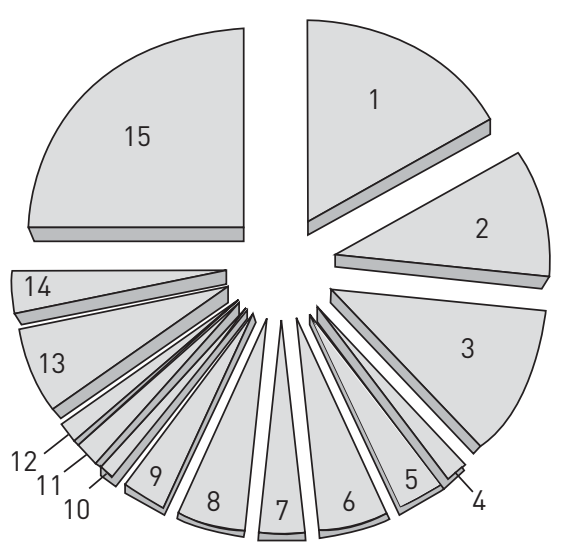

1 Pseudomonas aeruginosa

Total $\mathbf{n}=60^{\#}$

Multidrug-resistant Pseudomonas aeruginosa $6(10)$

3 Methicillin-sensitive Staphylococcus aureus 7 (12)

4 Methicillin-resistant Staphylococcus aureus 1 (2)

5 Escherichia coli 2 (3)

6 Serratia marcescens 3 (5)

7 Enterobacter species 2 (3)

8 ESBL-producing Enterobacteriaceae 3 (5)

9 Proteus species $2(3)$

10 Klebsiella pneumoniae 1 (2)

11 Morganella morganii 1 (2)

12 Stenotrophomonas maltophilia $1(2)$

13 Streptococcus pneumoniae 4 (7)

14 Aspergillus species 2 (3)

15 Other $15(25)$

FIGURE 3 Pathogens in lung transplant recipients that caused ventilator-associated tracheobronchitis. ESBL: extended-spectrum $\beta$-lactamase. ${ }^{\#}$ : polymicrobial in five cases. 


\begin{tabular}{|c|c|c|c|}
\hline & No infection & VAT & VAP \\
\hline Subjects $\mathrm{n}$ & 114 & 36 & 20 \\
\hline Mechanical ventilation days & $4(1-23.2)$ & $32(25-58)^{\#}$ & $47(31.2-113.5)^{\#}$ \\
\hline Time in ICU days & $8(5-27)$ & $35.5(28.2-64)^{\#}$ & $50.5(38-115)^{\#}$ \\
\hline Time in hospital days & $32(25.2-48.5)$ & $62.5(43.2-80)^{\#}$ & $67(45-126.2)^{\pi}$ \\
\hline ICU mortality & $6(5.2)$ & $3(8.3)^{+}$ & $8(40)^{9}$ \\
\hline Hospital mortality & 9 (7.9) & $6(16.6)^{+}$ & $10(50)^{\pi}$ \\
\hline
\end{tabular}

had recurring pulmonary sepsis. In 1991, Augustine et al. [34] identified gastrointestinal complications as a risk factor for respiratory infection in heart-lung transplant recipients. Vos et al. [35] found an association between bile acid aspiration and $P$. aeruginosa airway colonisation after lung transplantation. But, to our knowledge, the present study is the first to find a significant association between gastroparesis and VAP in the immediate post-lung transplant period. The trend we found of worse mortality in the patients with gastroparesis may be due to the higher incidence of VAP. Studies are needed on measures to prevent aspiration, such as fundoplication, continuous post-operative gastric suctioning, duodenal feeding and pro-kinetic drug-based nutrition protocols. Moreover, given the high incidence of multidrug-resistant organisms causing respiratory infections, investigations into the effects of selective digestive tract decontamination on this incidence would increase the currently available evidence in this field.

Diaphragmatic paresis is another repercussion of nerve injury during lung transplantation [36]. In agreement with previous reports [37], we found that it was associated with longer mechanical ventilation, and ICU and hospital stay. Patients with diaphragmatic paresis had more episodes of ventilator-associated respiratory infections, but the multivariate analysis showed that it was not an independent risk factor for VAP. In addition, in accordance with previous studies, there was no difference in mortality between patients with diaphragmatic paresis and those without the condition [36,37]. Further studies comparing patients with versus without diaphragmatic paresis would be of interest.

Also in accordance with previous studies $[10,38,39]$, pre-transplant colonisation was not associated with a higher incidence of ventilator-associated respiratory infection and patients with cystic fibrosis (despite the fact that they were all colonised, frequently by multidrug-resistant organisms) did not present more episodes of VAT or VAP than the rest of the population. The same was true for all the other underlying diseases. Mechanical ventilation prior to transplant was also not a significant risk factor for post-transplant pneumonia. All these findings are probably due to the adequate effect of antibiotic prophylaxis based on

FIGURE 4 Kaplan-Meier graph of 1-year survival in patients with and without post-lung-transplant ventilator-associated pneumonia (VAP). $\mathrm{p}<0.001$ via log rank test.

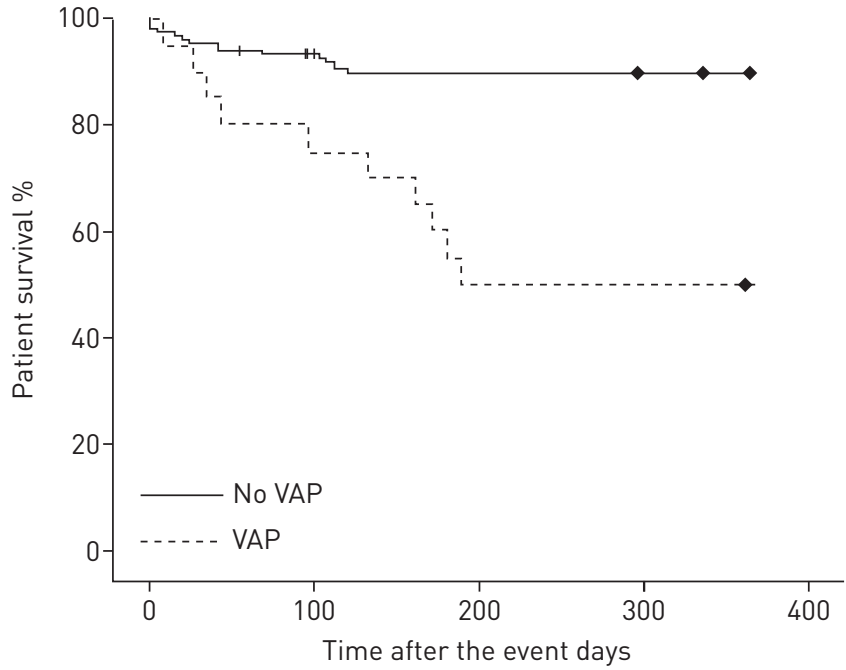


close microorganism surveillance before surgery. Also, Aspergillus was effectively prevented by nebulisation of amphotericin B.

The limitations of the present study are inherent to its retrospective nature. In addition, data derived from a single centre may limit the generalisation of the results to centres that may have substantially different management of lung transplant recipients. We did not evaluate the presence of gastro-oesophageal reflux in the patients with gastroparesis. However, the methods used to diagnose gastro-oesophageal reflux have only moderate sensitivity/specificity and can be invasive and expensive [40] so, similar to previous studies [16], we assumed that with the criteria used for the gastroparesis definition we would find an association with post-lung-transplant respiratory infections. Finally, the small sample size may have led to a type II error and may preclude some statistical comparisons. Nevertheless, the association between gastroparesis, VAP and outcomes was robust and has important clinical implications.

In conclusion, preventing VAP after lung transplant may improve survival. The association between gastroparesis and VAP may open up a new avenue for preventing pneumonia, thus reducing mortality and lowering costs. Treatment of ventilator-associated respiratory infections should cover multidrug-resistant bacteria. Preventing VAT may also decrease costs.

\section{Acknowledgements}

The Vall d'Hebron Lung Transplant Study Group investigators are as follows. Jordi Riera, Carolina Maldonado, Cristopher Mazo, Marina Garcia-de-Acilu, María Cubero, Berta Caralt, Mónica Ramírez-Martínez, Catalina Briceño and Jordi Rello (Critical Care Dept and Lung Transplant Unit, Vall d'Hebron University Hospital, Barcelona, Spain); Teresa Pont and Nuria Masnou (Critical Care Dept and Transplant Coordination Dept, Vall d'Hebron University Hospital); María Isabel Rochera, Montse Ribas and Daniel Ruiz (Dept of Anesthesiology and Lung Transplant Unit, Vall d'Hebron University Hospital); Juan Solé, Mercedes Canela, María Deu, Laura Romero, Iker López, Alberto Jaúregui and Javier Pérez (Dept of Thoracic Surgery and Lung Transplant Unit, Vall d'Hebron University Hospital); Antonio Roman, Carlos Bravo, Víctor Monforte, Manuel López-Meseguer and Cristina Berastegui (Dept of Pulmonology and Lung Transplant Unit, Vall d'Hebron University Hospital); and Joan Gavaldá and Oscar Len (Dept of Infectious Diseases and Lung Transplant Unit, Vall d'Hebron University Hospital).

\section{References}

1 Hosenpud JD, Bennett LE, Keck BM, et al. Effect of diagnosis on survival benefit of lung transplantation for end-stage lung disease. Lancet 1998; 351: 24-27.

2 Fishman JA. Infection in solid-organ transplant recipients. N Engl J Med 2007; 357: 2601-2614.

3 Aguilar-Guisado M, Givaldá J, Ussetti P, et al. Pneumonia after lung transplantation in the RESITRA cohort: a multi-center prospective study. Am J Transplant 2007; 7: 1989-1996.

4 Reid KR, McKenzie FN, Menkis $\mathrm{AH}$, et al. Importance of chronic aspiration in recipients of heart-lung transplants. Lancet 1990; 336: 206-208.

5 Robertson AG, Krishnan A, Ward C, et al. Anti-reflux surgery in lung transplant recipients: outcomes and effects on quality of life. Eur Respir J 2012; 39: 691-697.

6 Christie JD, Edwards LB, Kucheryavaya AY, et al. The Registry of the International Society for Heart and Lung Transplantation: 29th adult lung and heart-lung transplant report - 2012. J Heart Lung Transplant 2012; 31: 1073-1086.

7 Lockhart SR, Abramson MA, Beekmann SE, et al. Antimicrobial resistance among Gram-negative bacilli causing infections in intensive care unit patients in the United States between 1993 and 2004. J Clin Microbiol 2007; 45: 3352-3359.

8 Deusch E, End A, Grimm M, et al. Early bacterial infections in lung transplant recipients. Chest 1993; 104: $1412-1416$.

9 Mattner F, Fischer S, Weissbrodt $\mathrm{H}$, et al. Post-operative nosocomial infections after lung and heart transplantation. J Heart Lung Transplant 2007; 26: 241-249.

10 Dudau D, Camous J, Marchand S, et al. Incidence of nosocomial pneumonia and risk of recurrence after antimicrobial therapy in critically ill lung and heart-lung transplant patients. Clin Transplant 2014; 28: 27-36.

11 American Thoracic Society, Infectious Diseases Society of America. Guidelines for the management of adults with hospital-acquired, ventilator-associated, and healthcare-associated pneumonia. Am J Respir Crit Care Med 2005; 171: 388-416.

12 Palmer LB, Smaldone GC, Chen JJ, et al. Aerosolized antibiotics and ventilator-associated tracheobronchitis in the intensive care unit. Crit Care Med 2008; 36: 2008-2013.

13 Paterson DL. The epidemiological profile of infections with multidrugresistant Pseudomonas aeruginosa and Acinetobacter species. Clin Infect Dis 2006; 43 Suppl. 2:543-548.

14 Pitout JD, Laupland KB. Extended-spectrum $\beta$-lactamase-producing Enterobacteriaceae: an emerging public-health concern. Lancet Infect Dis 2008; 8: 159-166.

15 Matamis D, Soilemezi E, Tsagourias M, et al. Sonographic evaluation of the diaphragm in critically ill patients: technique and clinical applications. Intensive Care Med 2013; 39: 801-810.

16 Mentec H, Dupont H, Bocchetti M, et al. Upper digestive intolerance during enteral nutrition in critically ill patients: frequency, risk factors, and complications. Crit Care Med 2001; 29: 1955-1961.

17 Díaz-Ravetllat V, Greer M, Haverich A, et al. Significance of new lung infiltrates in outpatients after lung and heart-lung transplantation. Transpl Infect Dis 2014; 16: 359-368.

18 Craven DE, Lei $\mathrm{Y}$, Ruthazer R, et al. Incidence and outcomes of ventilator-associated tracheobronchitis and pneumonia. Am J Med 2013; 126: 542-549.

19 Bekaert M, Timsit JF, Vansteelandt S, et al. Attributable mortality of ventilator-associated pneumonia: a reappraisal using causal analysis. Am J Respir Crit Care Med 2011; 184: 1133-1139. 

meta-analysis of individual patient data from randomised prevention studies. Lancet Infect Dis 2013; 13: 665-671.

21 Stevens JP, Kachniarz B, Wright SB, et al. When policy gets it right: variability in U.S. hospitals' diagnosis of ventilator-associated pneumonia. Crit Care Med 2014; 42: 497-503.

22 Husain S, Mooney ML, Danziger-Isakov L, et al. A 2010 working formulation for the standardization of definitions of infections in cardiothoracic transplant recipients. J Heart Lung Transplant 2011; 30: 361-374.

23 Shahin J, Bielinski M, Guichon C, et al. Suspected ventilator-associated respiratory infection in severely ill patients: a prospective observational study. Crit Care 2013; 17: R251.

24 Nseir S, Di Pompeo C, Soubrier S, et al. Effect of ventilator-associated tracheobronchitis on outcome in patients without chronic respiratory failure: a case-control study. Crit Care 2005; 9: R238-R245.

25 Husain S, Chan KM, Palmer SM, et al. Bacteremia in lung transplant recipients in the current era. Am J Transplant 2006; 6: 3000-3007.

26 Berkowitz N, Schulman LL, McGregor C, et al. Gastroparesis after lung transplantation. Potential role in postoperative respiratory complications. Chest 1995; 108: 1602-1607.

27 Smith PC, Slaughter MS, Petty MG, et al. Abdominal complications after lung transplantation. J Heart Lung Transplant 1995; 14: 44-51.

28 Basseri B, Conklin JL, Pimentel M, et al. Esophageal motor dysfunction and gastroesophageal reflux are prevalent in lung transplant candidates. Ann Thorac Surg 2010; 90: 1630-1636.

29 Hadjiliadis D, Duane Davis R, Steele MP, et al. Gastroesophageal reflux disease in lung transplant recipients. Clin Transplant 2003; 17: 363-368.

30 Blondeau K, Mertens V, Vanaudenaerde BA, et al. Gastro-oesophageal reflux and gastric aspiration in lung transplant patients with or without chronic rejection. Eur Respir J 2008; 31: 707-713.

31 Cantu E 3rd, Appel JZ 3rd, Hartwig MG, et al. Maxwell Chamberlain Memorial Paper. Early fundoplication prevents chronic allograft dysfunction in patients with gastroesophageal reflux disease. Ann Thorac Surg 2004; 78: $1142-1151$.

32 Abbassi-Ghadi N, Kumar S, Cheung B, et al. Anti-reflux surgery for lung transplant recipients in the presence of impedance-detected duodenogastroesophageal reflux and bronchiolitis obliterans syndrome: a study of efficacy and safety. J Heart Lung Transplant 2013; 32: 588-595.

33 Akindipe OA, Faul JL, Vierra MA, et al. The surgical management of severe gastroparesis in heart/lung transplant recipients. Chest 2000; 117: 907-910.

34 Augustine SM, Yeo CJ, Buchman TG, et al. Gastrointestinal complications in heart and in heart-lung transplant patients. J Heart Lung Transplant 1991; 10: 547-555.

35 Vos R, Blondeau K, Vanaudenaerde BM, et al. Airway colonization and gastric aspiration after lung transplantation: do birds of a feather flock together? J Heart Lung Transplant 2008; 27: 843-849.

36 Sheridan PH Jr, Cheriyan A, Doud J, et al. Incidence of phrenic neuropathy after isolated lung transplantation. The Loyola University Lung Transplant Group. J Heart Lung Transplant 1995; 14: 684-691.

37 Ferdinande P, Bruyninckx F, Van Raemdonck D, et al. Phrenic nerve dysfunction after heart-lung and lung transplantation. J Heart Lung Transplant 2004; 23: 105-109.

38 Bonde PN, Patel ND, Borja MC, et al. Impact of donor lung organisms on post-lung transplant pneumonia. $J$ Heart Lung Transplant 2006; 25: 99-105.

39 Suhling H, Rademacher J, Greer M, et al. Inhaled colistin following lung transplantation in colonised cystic fibrosis patients. Eur Respir J 2013; 42: 542-544.

40 Hayat JO, Gabieta-Somnez S, Yazaki E, et al. Pepsin in saliva for the diagnosis of gastro-oesophageal reflux disease. Gut 2014 [In press DOI: 10.1136/gutjnl-2014-307049]. 